\title{
A FRACTAL MODEL OF THE UNIVERSE
}

\author{
Do Large Galaxies Interact by Quasar Exchange?
}

\author{
P. DRIESSEN \\ Av. Montjoie 24, Bte 6, 1050 Brussels, Belgium.
}

A fractal universe model has been proposed elsewhere (Driessen, 1991, 1994), based on the assumption of perfect self-similarity between some definite scales, and in particular between particles and galaxies. Its motivation rested essentially on two ideas: first, if striving for unification is justified, then the fractal idea makes sense, for in this case one single theory of particles could encompass all self-similar structures at once. Secondly, it could be demonstrated that, provided some reasonable hypothesis were made about the structure of spiral galaxy cores, they came back to the same topological state after two complete revolutions (Driessen, 1991). These ideas combined suggested that spiral galaxies might correspond to electrons on the cosmic scale. The main test of the coherence of the fractal picture was then to discover if other galaxy types and quasars do find a place in this model. Now, a small group of dissident has sustained for years that QSOs (or at least a certain class of QSOs) are local objects emitted by galaxies. This class would contain very small $\left(\mathrm{d} \approx 0.3 \mathrm{kpc}\right.$ ), faint $\left(M_{V} \approx-12\right)$, and most often radio-quiet objects with large (non cosmological) excess redshift. The analogy between the two statements "electrons emit photons" and "galaxies emit QSOs" hinted that QSOs embody cosmic scale photons, and entails that galaxies experience quasar exchange interactions. It was claimed further that galaxy clusters reproduce atomic and molecular-like systems with one or a few central giant ellipticals (the cosmic scale nuclei) interacting via quasar exchange with their satellite spirals (the cosmic scale electrons). The flock of dwarf galaxies around large ellipticals and spirals would result from condensation of dusty gas clouds entrained by ejected QSOs.

The model is falsifiable in the sense that it yields several strong predictions: large galaxies should always be surrounded by QSOs and attached to them by filaments as those emerging from NGC4319 or NGC1097. We call these "virtual" QSOs because they are still tied to their progenitor and hence do not constitute fully separate objects. The situation presumably mimics the cloud of virtual photons surrounding each electron. If the filament exerts a strong restoring force, the fate of most "virtual" QSOs is to fall back onto the galactic core, a process that could be depicted by a self-energy Feynman diagram. Due to the successive recoils created by these quasi periodic ejections and recaptures, galaxies should be submitted to a kind of Zitterbewegung. Sometimes a virtual quasar should be captured by a colliding galaxy and this would represent an "electrodynamical" type of galactic interaction, as in the photon exchange diagrams. Furthermore, this quasar exchange interaction should exert a repulsive force between spirals, and an attractive one between spirals and giant ellipticals. This clearly would have important consequences for the formation of clusters and larger structures, although they have not yet been worked out in detail. Finally, galaxies should display a quantum behaviour.

The supporting observational evidence is scant but it does exist and will be discussed in another paper. Let us simply note here that there is strong evidence for ejection (see Arp, 1987 and references therein). In Arp's famous system for example, an undulating filament emerging from the core of NGC4319 is directed 
toward MK205 (Sulentic, 1983). In fact I pointed out that the situation may be more complex than suspected hitherto. Because the halo of MK205 is about 10 times larger than those of other QSOs found very close to galaxies, I hypothesized that MK205 is not a quasar, but a Seyfert galaxy exchanging quasars with NGC4319 (Driessen, 1994). In that respect it would be interesting to investigate if the red companion 3" NNE of MK205 (Stockton et al., 1979) could be such an object. Interestingly, recent ROSAT X-ray observations (Arp, these proceedings) revealed compact X-ray sources connected to MK205 by lower surface brightness X-ray filaments. Three of the compact sources turned out to be QSOs of much higher redshift than MK205. This implies QSO ejection from MK205 and would, in my model, represent the virtual QSOs surrounding MK205. As for QSO absorption by a colliding galaxy, there is as yet no direct evidence but close inspection of the plates 103-4-5 of Arp's atlas (Arp, 1966) show interacting galaxies (spirals and ellipticals) whose cores are connected by extremely long, thin and straight filaments of unvarying width. These characteristics do not fit well with those expected from interaction plumes. In our model the filament connects the black-hole core of the parent galaxy to the core of the QSO which is currently swallowed by the receiving galaxy. Thus the filament appears as a direct connection between the two galaxies, and this illustrates a one-quasar exchange diagram. Allowing to the model, giant ellipticals also interact, and in this respect, it should be remembered that Arp has discovered that the jet in M87 points toward M84, another giant in the Virgo cluster (Arp, 1968).

Once the model is accepted as a working hypothesis, scaling factors can be calculated. Four determinations of the length scaling factor do not diverge for more than a factor 10 from the median which is $\Lambda_{L} \approx 2.410^{34}$. Unfortunately, only one determination of the time scaling factor was possible and it yielded $\Lambda_{T} \approx 3.110^{36} \mathrm{yr}$. The ratio gives the velocity scaling factor $\Lambda_{V}=\Lambda_{L} / \Lambda_{T} \approx 1 / 127$ which incidentally falls closes to $\alpha \approx 1 / 137$ (this may not be $c$. simple coincidence). It implies that free quasars should move at the constant speed $C=c \Lambda_{V} \approx 2200 \mathrm{~km} \mathrm{~s}^{-1}$ with respect to the cosmic background radiation. This would represent the strongest possible deviation from the Hubble flow for any galactic scale object. The quantum of angular momentum is found to be $H \approx 2.210^{77} \mathrm{gcm}^{2} \mathrm{~s}^{-1}$ which is much larger than the values computed for spirals with truncated rotation curves, so that the missing angular momentum is quite large as could be expected. A rough calculation shows that a value as high as $J=H / 2$ could result from a dark matter halo rotating at the asymptotic constant velocity. The strength of the quasar exchange force between two spiral galaxies is measured by the quantity $E^{2}=3.7910^{83} \mathrm{~g} \mathrm{~cm}^{3} \mathrm{~s}^{-2}$ which can be interpreted as the square of the spiral galaxy "charge". Now, the gravitational constant scales with dimensions so that distinct "constants" must be defined for each self-similar scale. We find new values of order $G \approx 710^{-12} \mathrm{~cm}^{3} \mathrm{~g}^{-1} \mathrm{~s}^{-2}$ on the galactic scale and $g \approx 510^{30} \mathrm{~cm}^{3} \mathrm{~g}^{-1} \mathrm{~s}^{-2}$ on the particle scale. Using $G$ and the mass of the typical spiral, it can be shown that the quasar exchange force is greater than the gravitational interaction. The value of $g$ is of the same order as that proposed by Oldershaw (1987) in a somewhat similar fractal model. Using $g$ together with $\hbar$ and $c$ in Planck's units furnishes a Planck mass very close to the proton mass, a Planck length close to the proton Compton wavelength and a Planck time close to the proton Zitterbewegung characteristic time. Similarly, when $G, H$ and $C$ are inserted into Planck's units, the latter fall close to characteristic quantities associated with giant ellipticals (our cosmic scale nuclei). It is clearly a very good point for the model that Planck's unruly values here reduce to those familiar quantities.

\section{REFERENCES}

Arp, H. (1966) Atlas of Peculiar Galaxies, Astrophysical Journal Suppl. Series 14, 1.

Arp, H. (1968) Publication of the Astronomical Society of the Pacific 80, 129.

Arp, H. (1987) Quasars, Redshift and Controversies, Interstellar Media, Berkeley.

Driessen, P. (1991) A Fractal Space-Time Model for Spinning Structures, unpublished.

Driessen, P. (1994) A Fractal Universe Model, submitted to Astrophysics and Space Science.

Oldershaw, R.L. (1987) Astrophysical Journal 322, 34.

Stockton, A.N., Wyckoff S., and Wehinger P.A. (1979) Astrophysical Journal 231, 673.

Sulentic, J.W. (1983) Astrophysical Journal 265, L49. 